\title{
Bridging length scales in colloidal liquids and interfaces from near-critical divergence to single particles
}

\author{
C. PATRICK ROYALL ${ }^{1,2 *}$, DIRK G. A. L. AARTS ${ }^{3}$ AND HAJIME TANAKA ${ }^{1 *}$ \\ ${ }^{1}$ Institute of Industrial Science, University of Tokyo, 4-6-1 Komaba, Meguro-ku, Tokyo 153-8505, Japan \\ ${ }^{2}$ School of Chemistry, University of Bristol, Bristol, BS8 1TS, UK \\ ${ }^{3}$ Physical and Theoretical Chemistry Laboratory, University of Oxford, South Parks Road, Oxford, 0X1 3QZ, UK \\ *e-mail: paddy@iis.u-tokyo.ac.jp; tanaka@iis.u-tokyo.ac.jp
}

Boiling and condensation are among the best recognized phase transitions of condensed matter. Approaching the critical point, a liquid becomes indistinguishable from its vapour, the interfacial thickness diverges and the system is dominated by long-wavelength density fluctuations ${ }^{1}$. Long wavelength usually means hundreds of particle diameters, but here we consider the limits of this assumption, using a mesoscopic analogue of simple liquids, a colloid-polymer mixture ${ }^{2,3}$. We simultaneously visualize both the colloidal particles and near-critical density fluctuations, and reveal particle-level images of the critical clusters and liquid-gas interface. Surprisingly, we find that critical scaling does not break down until the correlation length approaches the size of the constituent particles, where there is a smooth transition to non-critical classical behaviour. Our results could provide a framework for unifying the disparate particle and correlation length scales, and bring new insight into the nature of the liquid-gas interface and the limit of the critical regime.

Among the simplest pictures of two different phases is that of a magnet, whose sites can only point up or down, and do so depending solely on their neighbours. This Ising model in fact describes a general class of phase transitions ${ }^{1}$, from the original ferromagnetic transition in magnets to phase separation in alloys, binary liquids, emulsions and polymers, and the liquidgas transition in simple liquids and colloid-polymer mixtures. Although we shall consider the latter two cases, this work has implications for many systems that belong to the same universality class. Close to the critical point, many physical properties such as the bulk correlation length, $\xi_{\mathrm{B}}$, are described by simple scaling, such as $\xi_{\mathrm{B}}=\xi_{\mathrm{B}}^{0} \varepsilon^{-\nu}$, where $\xi_{\mathrm{B}}^{0}$ is the bare correlation length (or critical amplitude) and $\varepsilon=\left(T-T_{\mathrm{C}}\right) / T_{\mathrm{C}}$ is a reduced temperature where $T$ is the temperature and $T_{\mathrm{C}}$ is the critical temperature. The critical exponent $v=0.5$ according to mean-field theory, and very close to the critical point there is a crossover to $v=0.63$, 'three-dimensional (3D) Ising universality" ${ }^{\text {. We shall }}$ refer to the entire scaling regime, both mean field and 3D Ising as the critical region. Although criticality is well understood, the point at which it breaks down has so far received relatively little attention. Conventional theory of critical phenomena assumes a large separation in length scales, that is, that the correlation length is decoupled from other length scales. This length-scale separation lies at the heart of critical universality, implying that the microscopic details are irrelevant. Whereas length-scale coupling has been considered in complex fluids with much longer length scales, such as polymer ${ }^{5}$ and ionic ${ }^{6}$ solutions, we consider here a more general limit: the case that the correlation length approaches that of the constituent particles. Although it is difficult to approach this limit experimentally in molecular systems, a colloidpolymer mixture provides straightforward access to arbitrary effective temperatures.

Adding polymer to a colloidal suspension can induce a fluidfluid demixing widely accepted to be a larger scale analogue of liquid-gas coexistence in simple liquids, see the phase diagram in Fig. 1e, inset. The coexisting phases are a colloidal gas (rich in polymer, poor in colloids) and a colloidal liquid (rich in colloids, poor in polymer) ${ }^{7}$ whose structure resembles that of simple liquids ${ }^{8}$. Demixing stems from the entropy-driven attraction between the colloids, mediated by the polymers ${ }^{2,3}$. Under the assumption of ideal polymer behaviour, the interaction strength is proportional to the polymer concentration in a connected reservoir. We confirm that for our system, $1 / C_{\mathrm{P}}$ can reasonably be treated as the effective temperature (see the Supplementary Information). The results of some experimental studies notwithstanding ${ }^{9}$, previous work on colloid-polymer mixtures shows 3D Ising behaviour very close to criticality ${ }^{10,11}$. We therefore regard colloidpolymer mixtures as a mesoscopic model of simple liquids. Note that the explicit determination of 3D Ising criticality would be challenging for our system, which is optimized for the noncritical limit (see the Methods used on our samples and also Supplementary Information on the limit of the approach to the critical point). To explore 3D Ising criticality, we would require $\varepsilon \sim 10^{-5}$. This corresponds to a length scale of millimetres for our system of micrometre-sized colloids. Such a length scale cannot practically be accessed with a microscope; furthermore, macroscopic-scale experiments inevitably suffer from various sources of non-ideality such as gravity and confinement. In other words, for microscopic molecules, true criticality leads to mesoscopic length scales; for mesoscopic colloids, it leads to macroscopic length scales. In our experiments, thus, the lower bound of $\varepsilon$ is limited to $10^{-3}$, but we can access the high-temperature 'hard-sphere' limit $(\varepsilon \rightarrow \infty)$ simply by not adding polymer. 

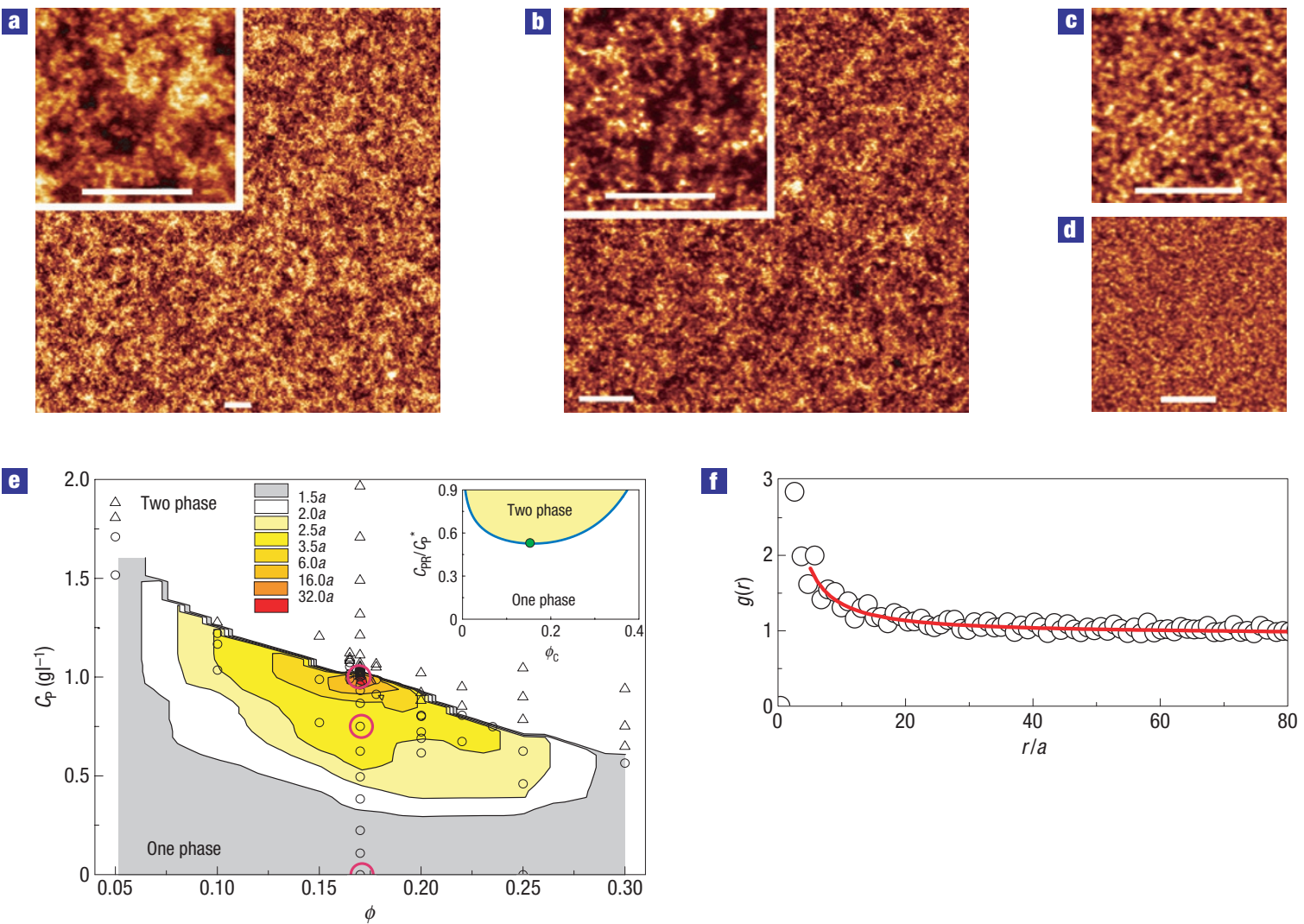

Figure 1 Near- and far-critical behaviour in the small-colloid system. a, Near-critical, $C_{\mathrm{p}}=1.057 \mathrm{gl}^{-1}$, showing single-particle-level detail and larger fluctuations. b. Small critical-like fluctuations, $C_{\mathrm{p}}=0.886 \mathrm{gl}^{-1}$. c,d, Non-critical, $C_{\mathrm{p}}=0$. Scale bars $=10 \mu \mathrm{m}$ and $\phi=0.17$ in all images. Polymers are not imaged. The images are ' $2 \mathrm{D}$ ' slices through the 3D sample. e, Contour plot of the correlation length overlaid with the phase diagram. Triangles are two-phase; circles are one-phase. The contour plot is interpolated between state points. The inset shows the polymer reservoir representation, which is directly analogous to simple fluids, calculated from theory ${ }^{25}$. The red circles in $\mathbf{e}$ show the state points pictured in a-d. f, Radial distribution function, fitted with the Ornstein-Zernike correlation function (red line) $C_{\mathrm{p}}=1.057 \mathrm{gl}^{-1}$.

We used two colloid-polymer mixtures. The small colloids (radius $a=230 \mathrm{~nm}$ ) allowed us to approach criticality to $\varepsilon \sim 10^{-3}$, whereas the larger colloids $(a=650 \mathrm{~nm})$ permitted full 3D resolution of the colloid coordinates ${ }^{12}$, see the Methods section. Confocal micrographs of the small-particle system are shown in Fig. 1. Near-critical behaviour, $C_{\mathrm{P}}=1.057 \mathrm{gl}^{-1}$, $\varepsilon=(2.7 \pm 1) \times 10^{-3}$, is illustrated in Fig. 1a. Note the multilevel information, both single-particle detail (Fig. 1a, inset) and bulk-like near-critical fluctuations (Fig. 1a). Relatively far from criticality, $C_{\mathrm{P}}=0.886 \mathrm{gl}^{-1}, \varepsilon=0.195 \pm 0.001$, the appearance is similar, Fig. 1b, although we have rescaled the image compared with the near-critical case. Non-critical behaviour is shown in Fig. $1 \mathrm{c}, \mathrm{d}, C_{\mathrm{P}}=0$, where only the large-scale single-particle-level image shows significant structure. Determination of the correlation length is described in the Methods section and shown in Fig. 1f. We plot the correlation length as a function of colloid and polymer concentration in Fig. 1e. Close to criticality, there is, as expected, a strong increase in the correlation length, shown in red. However, the subject of this work is the range over which the effects of critical fluctuations may be observed. As can be seen from Fig. 1e, it seems reasonable to say that this range covers a considerable region of the phase diagram.

We plot $\xi_{\mathrm{B}}(\varepsilon) / \xi_{\mathrm{B}}^{0}=\varepsilon^{-v}$ in the 'lambda-plot' in Fig. 2a and the $\log -\log$ plot in the inset for both experimental systems considered, along with the computer simulation data for an Asakura-Oosawa (AO) colloid-polymer mixture and a Lennard-Jones (LJ) model simple fluid (see the Methods section). The main observation here mirrors that of the contour plot in Fig. 1e, that the region where critical effects appear is large, extending to $\varepsilon \approx 1$ in all cases. Whereas deviations from critical scaling are small in the 'lambda-plot', the log-log plot (Fig. 2a, inset) shows a clear limit to critical scaling, on which the experimental data and $\mathrm{AO}$ simulation coincide. Near criticality, $\xi_{\mathrm{B}}$ follows the critical scaling $\xi_{\mathrm{B}}=\xi_{\mathrm{B}}^{0} \varepsilon^{-v}$ where we have redefined the reduced temperature for colloidpolymer mixtures $\varepsilon=\left(C_{\mathrm{P}}-C_{\mathrm{P}}^{C}\right) / C_{\mathrm{P}}$. At the level of this work, the value of the critical exponent, $v$, is not known to high accuracy. We obtain values of $v \approx 0.50$ when fitting the one-phase fluid data for the large and small particle. $\xi_{\mathrm{B}}$ starts to deviate from the scaling and approaches a constant value for $\varepsilon \gtrsim 1$. Our data are well fitted, including both critical and non-critical limits, by the following relation:

$$
\xi_{\mathrm{B}}\left(C_{\mathrm{P}}\right)=\xi_{\mathrm{B}}^{0}\left[\left(C_{\mathrm{P}}^{C}-C_{\mathrm{P}}\right) / C_{\mathrm{P}}^{C}\right]^{-v},
$$

where, compared with the usual relation, we substitute $C_{\mathrm{P}}^{C}$ by $C_{\mathrm{P}}$ in the denominator. This is equivalent to replacing $T$ by $T_{\mathrm{C}}$ in the conventional temperature notation, recovering the usual relation at small reduced temperature ${ }^{4}$. This replacement apparently saves the behaviour of the usual relation $\left(\xi_{\mathrm{B}} \rightarrow 0\right)$ in the non-critical classical limit $(\varepsilon \rightarrow \infty)$, although its validity needs to be verified. We also found $\xi_{\mathrm{B}+}^{0}=(1.28 \pm 0.4) a$ for the small colloids, and $\xi_{\mathrm{B}+}^{0}=(1.35 \pm 0.2) a$ and $\xi_{\mathrm{B}-}^{0}=(0.66 \pm 0.2) a$ for the large colloids, 

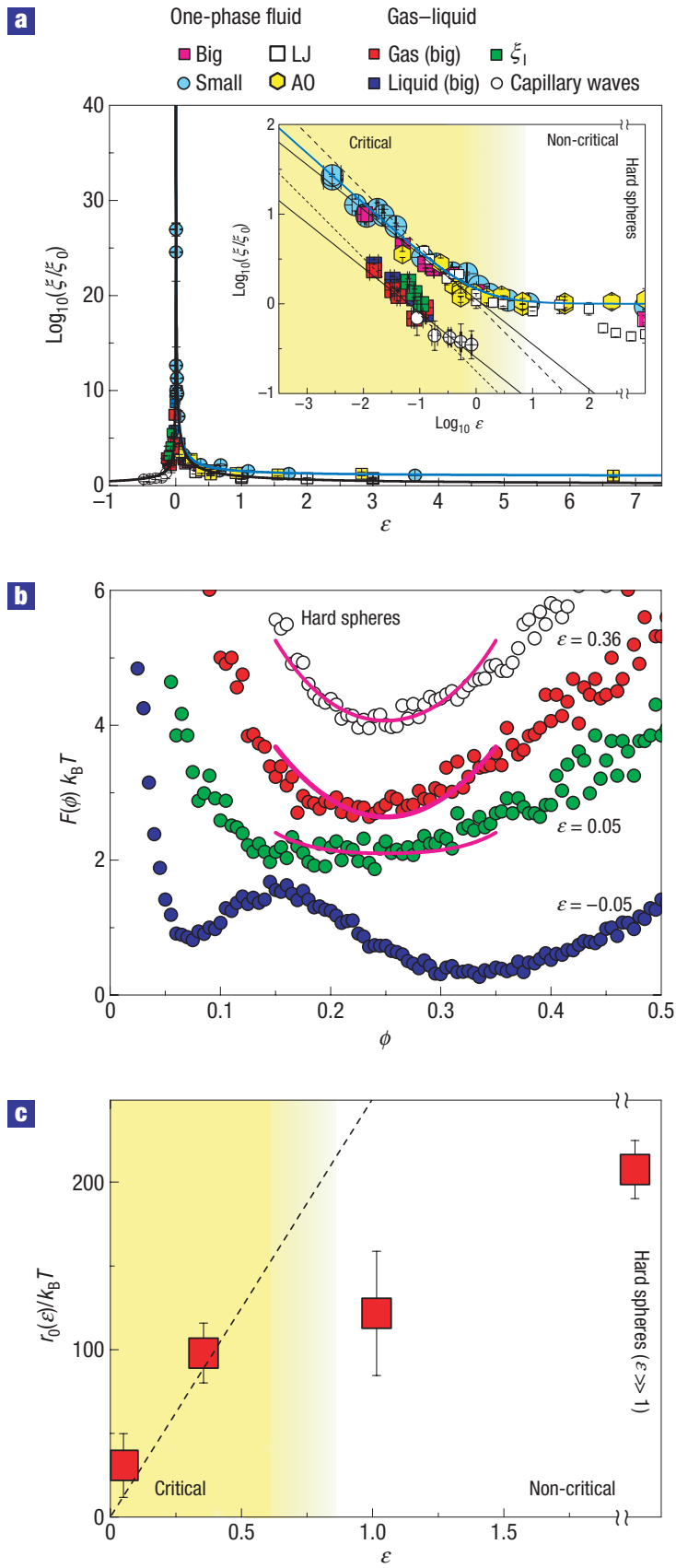

Figure 2 Near- and far-critical behaviour of correlation length, interfacial thickness and Helmholtz free energy. a, $\lambda$-plot of the correlation length, $\xi$, scaled by the bare correlation length, $\xi_{0}$. Experimental data are obtained from radial distribution function fitting (Fig. 1f), except $\xi_{1}$ defined in equation (2). Big and small refer to $a=650 \mathrm{~nm}$ and $a=230 \mathrm{~nm}$ colloids respectively. The black lines show $v=0.5$, dotted $v=0.63$, and the blue line is equation (1) with $v=0.54$. Inset: Same data on a log plot. Two-phase data offset by -0.5 for clarity. $\mathbf{b}$, Helmholtz free energy for the reduced temperatures; the pink lines are fits from Landau-Ginzburg theory (see the text). Radius $a=650 \mathrm{~nm}$. c, $r_{0}$ as a function of $\varepsilon$. The line is a linear fit to the data for $\varepsilon<0.4$, vanishing at the critical point. The shaded regions are guides to the eye. The error bars denote one standard deviation.

where + and - refer to the one-phase fluid and two-phase region respectively. This leads to a critical amplitude ratio of $\xi_{\mathrm{B}+}^{0} / \xi_{\mathrm{B}-}^{0}=1.9 \pm 0.3$, which is comparable to the theoretical value
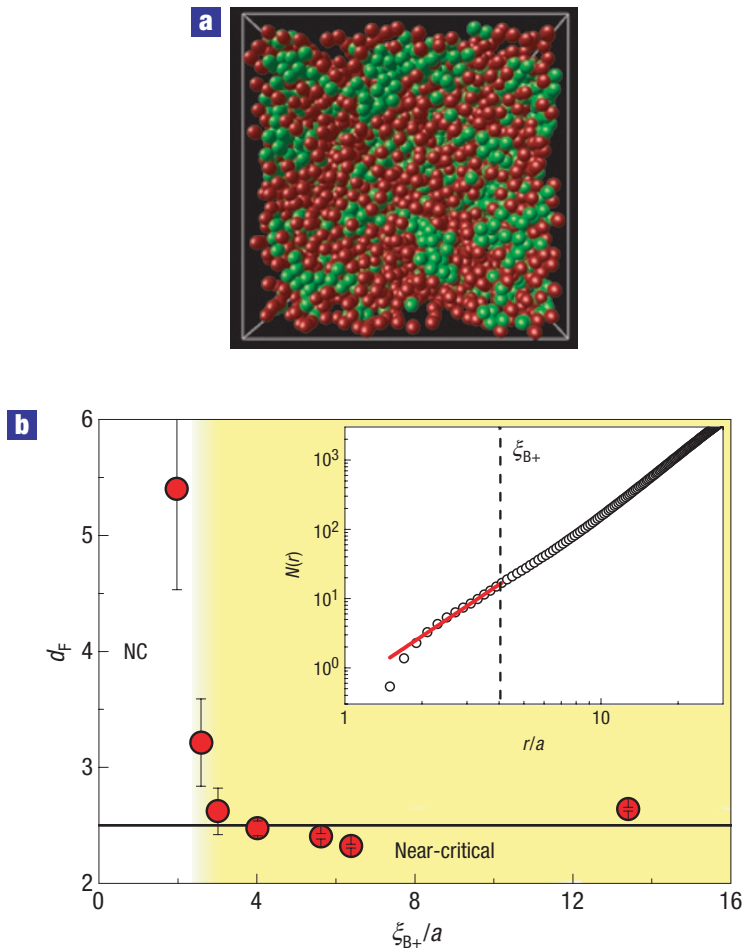

Figure 3 The fractal dimension of near-critical density fluctuations.

a, Near-critical density fluctuations at $\varepsilon=0.125 \pm 0.02$. The green particles belong to dense clusters, and red to dilute regions. $\mathbf{b}$, The fractal dimension of critical clusters as a function of the correlation length. The clusters are self-similar, except for $\xi_{\mathrm{B}+}<3 a$. The solid line is $d_{\mathrm{F}}=2.5$. Here, we use $a=650 \mathrm{~nm}$ colloids. The shaded regions are a guide to the eye. NC denotes non-critical. The error bars denote one standard deviation. Inset: Coordinate count as a function of $r / a$, fitted with the red line to yield a fractal dimension close to 2.5. The dashed line denotes $r=\xi_{\mathrm{B}+}, \varepsilon=0.125 \pm 0.02$.

(1.93 for 3D Ising and 1.41 for mean-field theory $)^{1}$. In Fig. 2a, we scaled the AO simulation data by $(0.81 \pm 0.05) a$, the mean value of $\xi$ for which $\varepsilon>1$, whereas the LJ results were unscaled ( $\xi_{0}$ set to $a$ ). Note the deviations at very high temperature $(\varepsilon \gtrsim 300)$ where the LJ repulsion 'softens'.

Dividing the sample into cells containing precisely one colloid, using the Voronoi construction ${ }^{13}$, allows us to determine $P(\phi)$, the probability distribution of the colloid volume fraction (1/cell volume), where $\phi$ is the colloid volume fraction. As the Helmholtz free energy may be defined as $F(\phi)=-k_{\mathrm{B}} T \ln (P(\phi))$, where $k_{\mathrm{B}}$ is Boltzmann's constant, we can directly measure $F(\phi)$ (Fig. $2 \mathrm{~b})^{14}$. With no polymer (white circles), the distribution has a single welldefined free-energy minimum. On adding polymer, approaching the critical point, the curve broadens, and eventually, in the two-phase region (blue circles) splits into two minima, one corresponding to gas and one to liquid. Although we cannot assume a priori that phenomenological coarse-grained theories such as Landau-Ginzburg will hold in this case, it is nevertheless possible to compare our experimental results. We analyse the curvature of the free energy at $\phi_{\mathrm{C}}, r_{0}$ (see the Supplementary Information). Figure 2c indicates that $r_{0}$ is approximately proportional to $\varepsilon$ for $\varepsilon<0.4$, in accord with Landau-Ginzburg theory. This range of $\varepsilon$ is similar to the range over which the correlation length obeys critical scaling.

The Voronoi construction also yields the fractal dimension of the critical clusters. Here, we select the densest $50 \%$ of the 


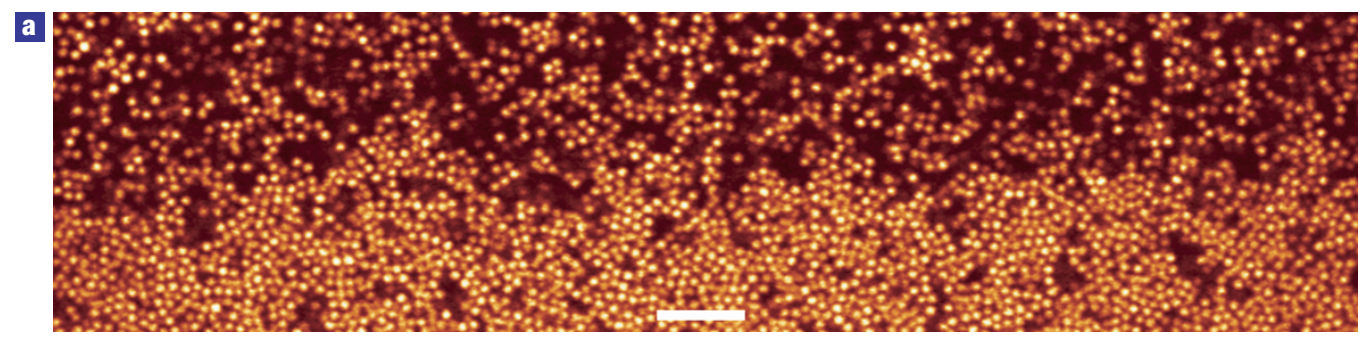

b

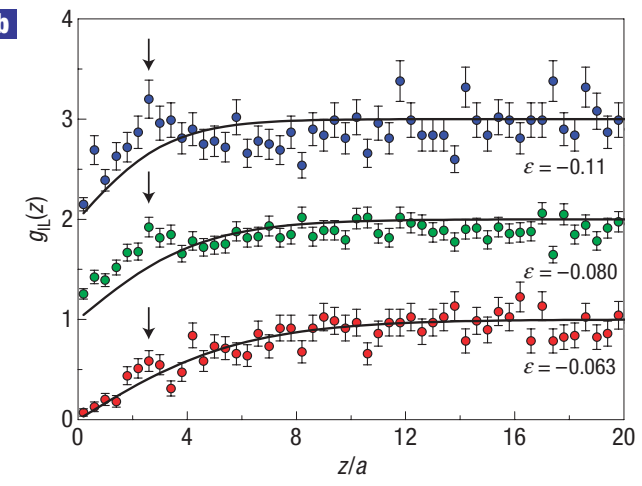

c

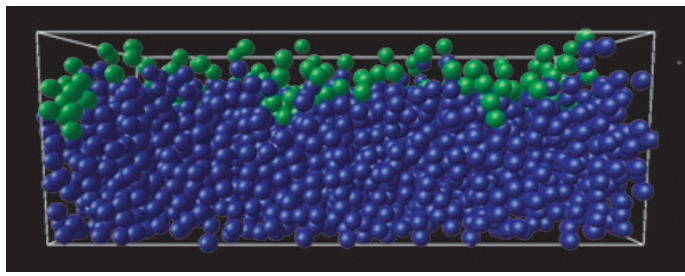

d

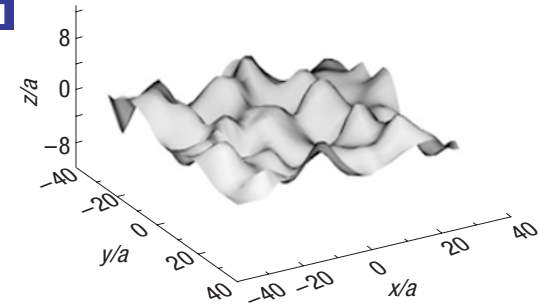

Figure 4 The liquid-gas interface at the single-particle level. a, Confocal micrograph of the liquid-gas interface at the single-particle level, $\varepsilon=-0.15$. Bar $=10 \mu \mathrm{m}$. b, Layering on the interface. The arrows show the first layer, which diminishes approaching criticality. The solid lines are fits from equation (2). The data are offset for clarity. Here, we use $a=650 \mathrm{~nm}$ colloids. The error bars denote one standard deviation. c, 3D representation of the interface at the single-particle level. Blue particles are liquid, green are interface. $\mathbf{d}$, The fitted interface. A quintic function fitted to the green particles in c.

colloids, revealing the critical density fluctuations, as shown in Fig. 3a. Considering only these dense particles, the number of particles as a function of distance, $r$, from each coordinate then directly leads to $N(r) \propto r^{d_{\mathrm{F}}}$, where $d_{\mathrm{F}}$ is the fractal dimension. We expect $d_{\mathrm{F}}=2.5$ (ref. 1 ), but pioneering work on binary liquids was hampered by limited resolution, such that $d_{\mathrm{F}}$ was measured as 2.8 (ref. 15). Here, we fit $N(r)$ from the single-particle level to the bulk correlation length $\xi_{\mathrm{B}}$, as shown by the red line in Fig. 3b, inset, and obtain fractal dimensions close to 2.5 across a wide range of polymer concentrations, see Fig. $3 \mathrm{~b}$. This provides evidence that the geometric characteristics of critical fluctuations (self-similarity), which are central to critical universality, can be observed even at length scales approaching the single-particle limit. For very short distances, the first peak of $g(r)$, that is, the first shell, leads to a fractal dimension greater than three, reflecting local structuring close to contact, and self-similarity breaks down. The same length scale follows from the breakdown of the critical scaling of $\xi_{\mathrm{B}}$.

We now investigate the relationship between the bulk correlation length, $\xi_{\mathrm{B}}$, and the intrinsic interfacial thickness, $\xi_{\mathrm{I}}$. The interface may be thought of either as a continuous density profile ${ }^{16}$ or a (step-like) local density profile dressed with thermal capillary waves $^{17,18}$. We measure the capillary waves and thus determine the interfacial tension, $\sigma$ (see the Supplementary Information). As the correlation length is proportional to $1 / \sqrt{\sigma}$ (ref. 1 ), this provides another measure of the scaling of the correlation length (see Fig. 2a). We also resolved the individual particles, Fig. 4a, which leads us to treat the liquid-gas interface at the single-particle level, following simulation work ${ }^{19}$ (see the Supplementary Information). Liquid particles are identified as those with at least four neighbours (Fig. 4c, blue particles). The top layer of liquid particles then defines the interface (Fig. 4c, green particles). These interface particles may be fitted with a smoothly varying interface (Fig. 4d), from which we calculated the fractal dimension of the interface to be $2.18 \pm 0.02$, for $\varepsilon=-0.042 \pm 0.003$, in an analogous way to the critical fluctuations.

In the layer below these interface particles, we find heightheight density correlations, $\rho(z)=\Sigma \delta\left(z-\left(z_{\mathrm{L}}-z_{\mathrm{I}}\right)\right)$, where $z_{\mathrm{I}}$ and $z_{\mathrm{L}}$ are the height coordinates of the interface and liquid particles respectively. Liquid particles are sampled from a column of radius $a$ below each interface particle. Figure $4 \mathrm{~b}$ shows $g_{\mathrm{IL}}(z)=(\rho(z)-\rho(0)) /(\rho(\infty)-\rho(0))$, which shows peaks around $3 a$ (arrows), indicating that there is some short-ranged structure below the interface. This reflects correlations in the liquid phase due to the microscopic definition of the interface, and is not necessarily connected to intrinsic layering ${ }^{20}$. Although we have followed a single-particle-level approach, we nonetheless note that it takes the $g_{\mathrm{IL}}(z)$ some time to rise to unity, indicating the intrinsic interfacial thickness. We are therefore motivated to fit these profiles in the spirit of van Der Waals theory ${ }^{16}$, excluding the few points comprising the peak around $3 a$ :

$$
g_{\mathrm{IL}}(z)=\tanh \left(-\frac{z}{2 \xi_{\mathrm{I}}}\right) .
$$

The results are plotted in Fig. 2a, showing a very similar result to the bulk correlation length. In other words, we succeeded in 
accessing the intrinsic interfacial profile, which is little affected by capillary waves.

In summary, we have demonstrated that both the intrinsic interfacial thickness and bulk correlation length have almost the same magnitude, and that critical scaling appears to have a limit where the correlation length falls to around the constituent particle size, for a colloid-polymer mixture. We observed the same behaviour with Monte Carlo simulations of a colloid-polymer mixture and an LJ model simple liquid at moderate temperatures. Thus, critical scaling may predict various physical properties, such as the density correlation length, interfacial tension and the free energy far from the critical point at least for colloid-polymer mixtures and some simple liquids. Furthermore, we find a smooth transition from critical to non-critical behaviour, which seems to be governed only by the size of the constituent particles themselves, representing hard-core repulsions. This not only raises questions as to why critical scaling works over such a range, but also has implications in its application to a wider range of materials and conditions than previously expected.

\section{METHODS}

\section{EXPERIMENTAL}

We used sterically stabilized polymethyl methacrylate colloids of $a=650$ (big) and $230 \mathrm{~nm}$ (small) radius and polydispersity $5 \%$, labelled with rhodamine ${ }^{21}$ and 1,1 dioctyadecyl-3,3,3,3-tetra methyl indocarbocyanine ${ }^{22}$ respectively. We used polystyrene with $M_{w}=3.0 \times 10^{7}$, which implies a polymer size of $164 \mathrm{~nm}$, and a polymer-colloid size ratio of around 0.25 for $a=650 \mathrm{~nm}$ and 0.7 for $a=230 \mathrm{~nm}$ in a theta solvent ${ }^{23}$. The larger colloids were dispersed in an almost density-matching solvent of 1:1.015:2.113 tetralin/cis-decalin/carbon tetrachloride by mass ${ }^{24}$. Comparison with free-volume theory ${ }^{25}$ suggests a strong degree of polymer swelling, perhaps even to $40 \%$, which, along with the polymer polydispersity $\left(M_{w} / M_{n}=1.3\right)$, should further stabilize the liquid-gas demixing $^{26}$. Demixing occurred in around $30 \mathrm{~min}$, and after around 1 day crystallites were nucleated at the walls of the container. This is distinct from fluid-crystal coexistence with smaller polymers ${ }^{7}$, when crystallization occurs immediately. The smaller colloids were dispersed in cis-decalin, and we saw no crystallization at all. Our simulations demonstrate (see Supplementary Information, Fig. S1) that, for ideal polymers, the assumption of proportionality between the polymer reservoir and the system is at least reasonable. Although the polymers used were not ideal, in the cis-decalin system, the experiments were carried out $10^{\circ} \mathrm{C}$ above the theta temperature, whereas in the case of the solvent mixture, the theta temperature was $>100^{\circ} \mathrm{C}$ lower. That we see no significant difference between the two systems suggests that polymer non-ideality is not important here.

To determine the critical point, we first found the critical colloid density, $\phi_{\mathrm{C}}$, by preparing suspensions at differing $\phi$, and for each we varied the polymer concentration (see the Supplementary Information).

\section{RADIAL DISTRIBUTION FUNCTION}

Pixel-level radial distribution functions, $g(r)$, were calculated from $2 \mathrm{D}$ images. We used 2D images because it was hard to collect data over the $100 \times 100 \times 100 \mu \mathrm{m}$ volume needed in the case of $3 \mathrm{D}$ images close to criticality. We note that in the case of the small particles, towards the hard-sphere limit, the measured value of the correlation length may be increased owing to the limited axial resolution of the microscope. However, we obtained similar relative values of $\xi$ for both systems, and conclude that such blurring has little effect in this case. We checked that there was no difference between correlation lengths extracted from 3D coordinate data, where we fitted a straight line to $\ln (r|g(r)-1|)$. We determined the correlation length from simulation data in this way also.

\section{MONTE CARLO SIMULATION}

We carried out standard Monte Carlo simulations at constant particle number, volume and temperature for an LJ system $\left.\left(u_{\mathrm{LJ}}\right)=4 w\left((\sigma / r)^{12}-(\sigma / r)^{6}\right)\right)$, where the interaction was truncated at $2.5 \sigma$ and shifted such that $u_{\mathrm{LJ}}(2.5 \sigma)=0$. We used 4,000 particles, equilibrated with up to $2 \times 10^{8}$ steps. The critical temperature was taken as $T_{\mathrm{C}}=1 / 4 w=1.1876$, and density as $\rho_{\mathrm{C}}=0.3197 \sigma^{-3}$ (ref. 27). We fixed the density, and varied the temperature. In the case of the colloid-polymer mixture, we used the AO mode ${ }^{28}$ of hard-sphere colloids and ideal polymers, which may not overlap with the colloids. The polymers were in chemical equilibrium with a reservoir at fixed concentration. We used 1,000 colloids and up to 5,000 polymers, and up to $10^{8}$ equilibration steps. We set the colloid-polymer size ratio to 0.8 and took the critical values of $C_{\mathrm{PR}}^{C} / C_{\mathrm{P}}^{*}=0.766, C_{\mathrm{P}}^{C} / C_{\mathrm{P}}^{*}=0.3562$ and $\phi_{\mathrm{C}}=0.134$ where $C_{\mathrm{P}}^{C}$ and $C_{\mathrm{PR}}^{C}$ are the critical polymer volume fractions in the system and reservoir respectively and $C_{\mathrm{P}}^{*}$ is the polymer overlap concentration ${ }^{28}$.

\section{Received 26 January 2007; accepted 27 June 2007; published 22 July 2007.}

\section{References}

1. Onuki, A. Phase Transition Dynamics (Cambridge Univ. Press, Cambridge, 2002).

2. Asakura, S. \& Oosawa, F. On interaction between two bodies immersed in a solution of macromolecules. J. Chem. Phys. 22, 1255-1256 (1955).

3. Vrij, A. Polymers at interfaces and interactions in colloidal dispersions. Pure Appl. Chem. $\mathbf{4 8}$ 471-483 (1976).

4. Kim, Y. C., Anisimov, M. A., Sengers, J. V. \& Luijten, E. Crossover critical behavior in the three-dimensional Ising model. J. Stat. Phys. 110, 591-609 (2003).

5. Anisimov, M. A., Kostko, F. A., Sengers, J. V. \& Yudin, I. K. Competition of mesoscales and crossover to theta-point tricriticality in near-critical polymer solutions. J. Chem. Phys. 123, 164901 (2005).

6. Narayanan, T. \& Pitzera, K. S. Critical phenomena in ionic fluids: A systematic investigation of the crossover behavior. J. Chem. Phys. 102, 8118-8131 (1997).

7. Poon, W. C. K. The physics of a model colloid-polymer mixture. J. Phys. Condens. Matter 14, R859-R880 (2002)

8. Moussaid, A., Poon, W. \& Soliva, M. Structure of marginal and fully-developed colloidal liquids. Phys. Rev. Lett. 82, 225-228 (1999).

9. Bodnar, I., Dhont, J. K. G. \& Lekkerkerker, H. N. W. Pretransitional phenomena of a colloid polymer mixture with static and dynamic light scattering. J. Phys. Chem. 100, 19614-19619 (1996).

10. Chen, B.-H., Payandeh, B. \& Robert, M. Order parameter and interfacial tension of a colloid-polymer system. Phys. Rev. E 62, 2369-2372 (2000)

11. Lo Verso, F., Vink, R. L. C., Pini, D. \& Reatto, L. Critical behavior in colloid-polymer mixtures: Theory and simulation. Phys. Rev. E 73, 061407 (2006).

12. van Blaaderen, A. \& Wiltzius, P. Real-space structure of colloidal hard-sphere glasses. Science 270, $1177-1179$ (1995)

13. Barber, C. B., Dobkin, D. P. \& Huhdanpaa, H. T. The quickhull algorithm for convex hulls. ACM Trans. Math. Software 22, 469-483 (1996).

14. Tanaka, H. \& Nishi, T. Direct determination of the probability distribution function of concentration in polymer mixtures undergoing phase separation. Phys. Rev. Lett. 59, 692-695 (1987).

15. Guenoun, P., Perrot, F. \& Beysens, D. Microscopic observation of order-parameter fluctuations in critical binary fluids: Morphology, self-similarity and fractal dimension. Phys. Rev. Lett. 63, 1152-1156 (1989)

16. van der Waals, J. D. The thermodynamic theory of capillarity under the hypothesis of a continuous variation of density, Vol. 20 (Verh. Konink. Akad. Weten. Amsterdam (sect. 1), 1983). English translation by Rowlinson, J. S., J. Stat. Phys. 20, 197-200 (1979).

17. Buff, F. P., Lovett, R. A. \& Stillinger, R. H. Interfacial density profile for fluids in the critical region. Phys. Rev. Lett. 15, 621-623 (1965).

18. Aarts, D. G. A. L., Schmidt, M. \& Lekkerkerker, H. N. W. Direct observation of thermal capillary waves. Science 304, 847-850 (2004).

19. Chacon, E. \& Tarazona, P. Intrinsic profiles beyond capillary wave theory: A Monte Carlo study. Phys. Rev. Lett. 91, 166103 (2003).

20. Magnussen, O. M., Ocko, B. M., Regan, M. J., Penanen, K. \& Pershan, P. S. X-ray reflectivity measurements of surface layering in liquid mercury. Phys. Rev. Lett. 74, 4444-4447 (1995).

21. Bosma, G. et al. Preparation of monodisperse, fluorescent PMMA-latex colloids by dispersion polymerization. J. Colloid Interface Sci. 245, 292-300 (2002).

22. Campbell, A. I. \& Bartlett, P. Fluorescent hard-sphere colloids for confocal microscopy. J. Colloid Interface Sci. 256, 325-330 (2002).

23. Vincent, B. The calculation of depletion layer thickness as a function of bulk polymer concentration. Colloids Surf. 50, 241-249 (1990).

24. de Hoog, E. H. A., Kegel, W. K., van Blaaderen, A. \& Lekkerkerker, H. N. W. Direct observation of crystallization and aggregation in a phase-separating colloid-polymer suspension. Phys. Rev. E 64, 021407 (2001).

25. Lekkerkerker, H. N. W., Poon, W. C. K., Pusey, P. N., Stroobants, A. \& Warren, P. B. Phase-behavior of colloid plus polymer mixtures. Europhys. Lett. 20, 559-564 (1992).

26. Fasolo, M. \& Sollich, P. Effects of polymer polydispersity on the phase behaviour of colloid-polymer mixtures. J. Phys. Condens. Matter 17, 797-812 (2005).

27. Wilding, N. Critical-point and coexistence-curve properties of the Lennard-Jones fluid: A finite-size scaling study. Phys. Rev. E 52, 602-611 (1995).

28. Vink, R. L. C. \& Horbach, J. Grand canonical Monte Carlo simulation of a model colloid polymer mixture: Coexistence line, critical behavior, and interfacial tension. J. Chem. Phys. 121, 3253-3258 (2004).

\section{Acknowledgements}

The authors are grateful to M. Anisimov, A. van Blaaderen, P. Bartlett, D. Bonn, D. Derks, B. Evans, J.-P. Hansen, R. Jack, H. Lekkerkerker, W. Poon, P. Pusey, A. Onuki, M. Schmidt, J. Sengers and R. Vink for valuable discussions and comments. This work was partially supported by a grant-in-aid from the Ministry of Education, Culture, Sports, Science and Technology, Japan. D.G.A.L.A. acknowledges the Van 't Hoff Laboratory for Physical and Colloid Chemistry, Utrecht, The Netherlands, and the Laboratoire de Physique Statistique of the Ecole Normale Supérieure, Paris.

Correspondence and requests for materials should be addressed to C.P.R. or H.T

Supplementary Information accompanies this paper on www.nature.com/naturephysics.

\section{Author contributions}

C.P.R. carried out the experiments and analysis, D.G.A.L.A. conducted preliminary experiments and H.T. supervised the project. All authors co-wrote the paper.

Competing financial interests

The authors declare no competing financial interests. 